\section{Another Tip Collecting Serial Ultrathin Sections}

I do quite a bit of serial sectioning and would like to offer e following advice:

1) Cut the sections with as small a face as possible. They an be as much as one millimeter wide, but try to make them omething like $0.1 \mathrm{~mm}$ high. This will allow for as many as 20 ections on a $2 \mathrm{~mm}$ slotted grid.

2) Get a bunch of locking tweezers, and from the time the rid is picked up until it is put into the microscope, do not put e grid down onto filter paper (as for a normal grid). So, after icking up the ribbon of sections, leave the grid in the tweezrs to dry, and when post-staining the grids, leave them in the veezers to dry. If the grids are put down onto filter paper, the ormvar may rupture.

3) Maintain a positive attitude, and if things aren't going our way; stop and come back to it another day (the blocks ill always be there for you, but if you get burned out you 'on't be there for the blocks).

Tim Schneider, Thomas Jefferson University Timothy.Schneider@mail.tju.edu

\section{Another Lens Cleaning Tip}

Here is an odd way that I thought up to clean oil from an ojective without using solvents that may attack lens cement.

Remove the lens from the scope. I usually like to view the contamination using a stereoscopic microscope. Wipe exess oil away - l like using Ross Optical Paper. Using a cotton oplicator wrapped in Ross optical paper, apply a small mount of Dawn dishwashing detergent. Gently work the surce to emulsify the oil into the detergent using the opticalapered applicator. Add a small amount of water if needed. o not allow fluids to go much beyond the lens area!

At this point, hold the lens vertical with the back focal ane pointing up. Apply a small amount of deionized water n the final lens element from the side of the objective to crete a hanging drop. I usually use a wash bottle or $10 \mathrm{cc}$ sygge. The surface tension of the water will create a small op around the optical surface. Start a stream of deionized ater flowing through the small drop to wash away the oil/ ater suspension. After about a minute, stop the water flow hile allowing a small drop of water to stay on lens. At this ne, blow the water off using $\mathrm{CO}_{2}$ or some other clean comressed gas. This will eliminate streaks. Upon inspection, if ontamination can still be seen, repeat the process and then e problem should be solved.

It's weird but it works.

Robert Fitton, Luther College fittonro@luther.edu

The "Whistle" Test

In a recent discussion on the Microscopy Listserver about oise interference in the TEM, I recalled what a service engieer told me about checking for noise interference. Every lechanism has a resonate frequency and a loud enough oise at that frequency will cause it to "buzz" or vibrate. The tage of the TEM can be quite susceptible to this. When look- ing at the image on the TEM through the binoculars at a magnification of 200,000 times or higher, and talking to a class around the TEM at the same time, the image may be seen vibrating or looking fuzzy at certain times while talking. I make it a point to tell users of the TEM not to talk while taking a photo, and not to take a photo while the noisy air compressor is on.

To pin this interference down more precisely, perform the $\lambda$ "whistle test": load a sample with a fine structure and sharp $九$ edges, go to fairly high magnification $(>200,000 X)$ and focus carefully. Then while watching the image carefully through the binocular viewer, whistle a low note and slowly go up in frequency. Look for the whistle note that causes the image to blur. If this is not seen, the stage is very stable and will probably not be bothered much by middle frequency sounds. If the phenomenon is seen, the troublesome frequencies will be known, and can be avoided or muffled, and as well, which noises to avoid during photos.

Mary Mager, University of British Columbia mager@interchange.ubc.ca
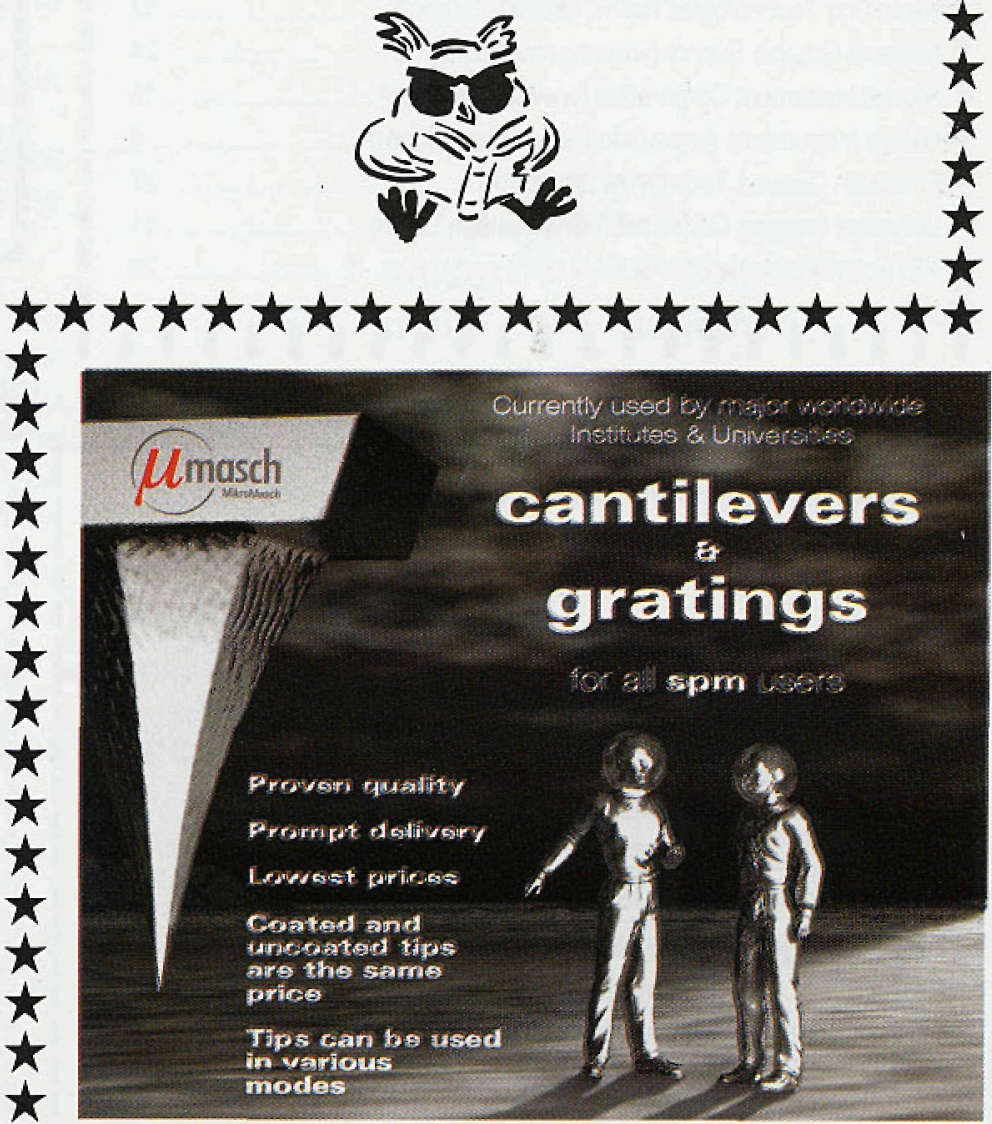

welcome to Spmtips.com

contracer:

K-TEK Imternational, Inc

1006 SW Reveliarid st

Forriarid, OF: 902128

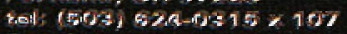

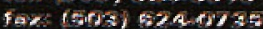

For pribing arid testirition

informations call Foll fres

sese-716agli 